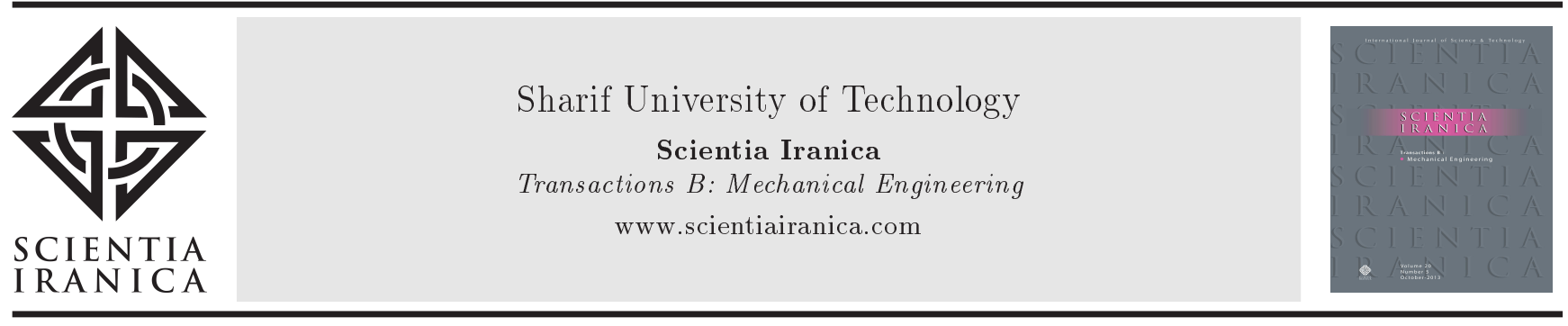

\title{
Study of peristaltic flow of magnetohydrodynamic Walter's B fluid with slip and heat transfer
}

\author{
A.A. Khan ${ }^{\mathrm{a}}$, H. Usman ${ }^{\mathrm{a}}$, K. Vafai ${ }^{\mathrm{b}}$ and R. Ellahi ${ }^{\mathrm{a}, \mathrm{c}, *}$ \\ a. Department of Mathematics \& Statistics, FBAS, IIUI, H-10, Islamabad, Pakistan. \\ b. Department of Mechanical Engineering, University of California, Riverside, USA. \\ c. Department of Mathematics, Faculty of Science, Taibah University, Madinah Munawwarah, Saudi Arabia.
}

Received 12 February 2015; received in revised form 24 October 2015; accepted 8 October 2016

\section{KEYWORDS}

Magnetohydrodynamics;

Peristaltic flow;

Walter's B fluid;

Heat transfer;

Slip condition;

Regular perturbation

method;

Analytical solutions.

\begin{abstract}
The purpose of this paper is to investigate the effects of magnetohydrodynamic peristaltic flow of Walter's B fluid in an inclined asymmetric channel under the influence of slip conditions. The effects of heat and mass transfer are also taken into account. Analytical solutions of nonlinear coupled equations are obtained by regular perturbation method. Graphs for different flow parameters of interest are sketched and analyzed. It is observed that the absolute value of shear stress and heat transfer coefficient decreases by increasing the magnetic parameter, whereas with the increase of magnetic parameter, concentration decreases. Opposite behavior has been noted for temperature and heat transfer coefficient at upper and lower walls against various values of Prandtl number, Eckert number, slip parameter, and material constant of Walter's B fluid. Oscillatory behavior of heat transfer coefficient is observed, which is due to propagation of peristaltic waves along the walls of the channel.

(C) 2016 Sharif University of Technology. All rights reserved.
\end{abstract}

\section{Introduction}

Peristaltic transport is a mechanism for mixing and transporting fluids, which is caused by a progressive wave of contraction and expansion travelling on the walls of the channel. The study of peristalsis has gained a distinct status amongst several researchers because of its various applications in physiological processes [1]. Moreover, effects of heat transfer have a significant role in peristalsis processes, such as oxygenation and hemodialysis. The properties of tissue can be pigeonholed through heat transfer analysis. Heat is either produced or locally inserted to monitor the thermal clearance rate [2-5]. Likewise, the effects of magnetohydrodynamics in peristalsis are very much noteworthy in magnetic therapy, arterial

*. Corresponding author.

E-mail addresses: e-mail: rellahi@engr.ucr.edu; and rahmatellahi@yahoo.com (R. Ellahi) flow, hyperthermia compressor, etc. Magnets could heat inflammations, ulceration, and several diseases of intestine and uterus. Recently, several studies have been made on the peristaltic motion of non-Newtonian fluids under different assumptions.

Furthermore, Beard and Walter [6] have proposed the constitutive equations for elastico-viscous fluids, which are formally known as Walters' B liquids. The Walters' B fluid model describes the performance of several polymeric liquids that come across in bioscience. It can tremendously predict the complex flow behavior of various industrial polymer solutions such as hydrocarbons, paints, etc. This model also incorporates the elastic properties of the fluids that are absolutely useful to understand certain extensional polymers. A very careful review of the literature divulges that amongst the viscoelastic fluids, the Walter's B model has gained very little attention so far. Few studies containing the relevant works on the topic can be mentioned by the attempts [7-12] and several therein. 
With all the aforementioned points in mind, one can clearly observe that no analysis for magnetohydrodynamic peristaltic flow of Walter's B fluid in the presence of heat and mass transfer under the influence of slip conditions is accorded in available literature yet. The courtesy in exploring the said topic is a motivating factor for this study. In fact, we intend to strengthen our efforts to understand the problems having more complicated nature. This is particularly in modeling of peristaltic flow of non-Newtonian fluids in an inclined asymmetric channel. The constitutive relationship of Walter's B fluid is used in the mathematical formulation of the problem. The resulting nonlinear governing equations are computed for the series solutions valid for small wave numbers and low Reynolds numbers. Graphs for magnetic parameter, slip parameter, heat transfer coefficient, Walter's B fluid parameter, Prandtl number, Eckert's number, Schmidt number, and Soret number are sketched and analyzed.

\section{Mathematical formulation of the problem}

Consider the Walter's B fluid through an inclined asymmetric channel of peristaltic transport having width $d_{1}+d_{2}$ such that the upper and lower walls are respectively at distances of $d_{1}$ and $d_{2}$ from the center line of the channel. The sinusoidal waves of different amplitudes and phases are moving along the channel walls with constant speed $c$. The upper and lower walls are maintained at temperatures $T_{0}$ and $T_{1}$, respectively. The upper and lower walls have different amplitudes $a_{i}(i=1,2)$ with phase difference $\phi(0 \leq \phi \leq \pi)$. The flow is considered in the direction of $\overline{\bar{X}}$-axis, whereas $\bar{Y}$-axis is taken normal to it. In the laboratory frame, we seek the velocity field of the form:

$$
\overline{\mathbf{V}}=[\bar{U}(\bar{X}, \bar{Y}, \bar{t}) \bar{V}(\bar{X}, \bar{Y}, \bar{t}) 0]
$$

in which $\bar{U}$ and $\bar{V}$ are the velocity components in the longitudinal and transverse directions correspondingly. The geometry of the channel walls $\bar{H}_{1}$ and $\bar{H}_{2}$ is defined as:

$$
\begin{aligned}
& \bar{H}_{1}(\bar{X}, \bar{t})=d_{1}+a_{1} \sin \left[\frac{2 \pi}{\lambda}(\bar{X}-c \bar{t})\right], \\
& \bar{H}_{2}(\bar{X}, \bar{t})=-d_{2}-a_{2} \sin \left[\frac{2 \pi}{\lambda}(\bar{X}-c \bar{t})+\phi\right] .
\end{aligned}
$$

Here, $\lambda$ is the wavelength and $\bar{t}$ is time.

The channel walls and magnetic field are inclined at angles $\omega$ and $\gamma$, respectively. A uniform magnetic field $B_{0}$ is applied in the transverse direction to the flow. The magnetic Reynolds number is taken small so that the induced magnetic field is neglected. Henceforth, the governing equations in the form of components are obtained as:

$$
\begin{aligned}
& \frac{\partial \bar{U}}{\partial \bar{X}}+\frac{\partial \bar{V}}{\partial \bar{Y}}=0 \\
& \rho\left(\frac{\partial}{\partial \bar{t}}+\bar{U} \frac{\partial}{\partial \bar{X}}+\bar{V} \frac{\partial}{\partial \bar{Y}}\right) \bar{U}=-\frac{\partial \bar{P}}{\partial \bar{X}}+\frac{\partial \bar{S}_{X X}}{\partial \bar{X}} \\
& +\frac{\partial \bar{S}_{X Y}}{\partial \bar{Y}}+\rho g \sin \omega-\sigma \boldsymbol{B}_{0}^{2} \cos \gamma(\bar{U} \cos \gamma-\bar{V} \sin \gamma), \\
& \rho\left(\frac{\partial}{\partial \bar{t}}+\bar{U} \frac{\partial}{\partial \bar{X}}+\bar{V} \frac{\partial}{\partial \bar{Y}}\right) \bar{V}=-\frac{\partial \bar{P}}{\partial \bar{Y}}+\frac{\partial \bar{S}_{X Y}}{\partial \bar{X}} \\
& +\frac{\partial \bar{S}_{Y Y}}{\partial \bar{Y}}-\rho g \cos \omega+\sigma \mathbf{B}_{0}^{2} \sin \gamma(\bar{U} \cos \gamma-\bar{V} \sin \gamma) \\
& \rho \xi\left(\frac{\partial}{\partial \bar{t}}+\bar{U} \frac{\partial}{\partial \bar{X}}+\bar{V} \frac{\partial}{\partial \bar{Y}}\right) T=k\left(\frac{\partial^{2} T}{\partial \bar{X}^{2}}+\frac{\partial^{2} T}{\partial \bar{Y}^{2}}\right)+\bar{\Phi}, \\
& \left(\frac{\partial}{\partial \bar{t}}+\bar{U} \frac{\partial}{\partial \bar{X}}+\bar{V} \frac{\partial}{\partial \bar{Y}}\right) C=D\left(\frac{\partial^{2} C}{\partial \bar{X}}+\frac{\partial^{2} C}{\partial \bar{Y}^{2}}\right) \\
& +\frac{D K_{T}}{T_{m}}\left(\frac{\partial^{2} T}{\partial \bar{X}^{2}}+\frac{\partial^{2} T}{\partial \bar{Y}^{2}}\right)
\end{aligned}
$$

The extra-stress tensor for Walter's B fluid is defined as [13]:

$$
\overline{\mathbf{S}}=2 \eta_{0} e-2 k_{0} \frac{\delta e}{\delta \bar{t}},
$$

in which:

$$
\begin{aligned}
& \frac{\delta e}{\delta \bar{t}}=\frac{\partial e}{\partial \bar{t}}=\mathbf{V} \cdot \nabla e-e \nabla \mathbf{V}-(\nabla \mathbf{V})^{T} e \\
& e=\nabla \mathbf{V}+(\nabla \mathbf{V})^{T} .
\end{aligned}
$$

The moving and fixed frames are related by the expressions:

$$
\bar{x}=\bar{X}-c \bar{t}, \quad \bar{y}=\bar{Y}, \quad \bar{u}=\bar{U}-c, \quad \bar{v}=\bar{V}, \quad \bar{p}=\bar{P} .
$$

The flow becomes steady in the wave frame $(\bar{x}, \bar{y})$ by opting a wave frame moving with velocity $c$ as that of travelling wave away from the laboratory frame $(\bar{X} \cdot \bar{Y})$.

Velocity components $u$ and $v$ in terms of stream function and dimensionless variables are defined by:

$$
\begin{aligned}
& u=\frac{\partial \psi}{\partial y}, \quad v=-\delta \frac{\partial \psi}{\partial x}, \quad x=\frac{\bar{x}}{\lambda}, y=\frac{\bar{y}}{d_{1}}, u=\frac{\bar{u}}{c} \\
& p=\frac{d_{1}^{2}}{\eta_{0} \lambda c} \bar{p}, \quad F r=\frac{c^{2}}{g d_{1}}, \quad h_{1}=\frac{\overline{h_{1}}}{d_{1}}, \quad h_{2}=\frac{\overline{h_{2}}}{d_{1}}
\end{aligned}
$$




$$
\begin{aligned}
& S=\frac{d_{1}}{\eta_{0} c} \bar{S}, \quad v=\frac{\bar{v}}{c}, \quad \delta=\frac{d_{1}}{\lambda}, \quad \operatorname{Re}=\frac{\rho c d_{1}}{\eta_{0}}, \\
& \kappa=\frac{k_{0} c}{\eta_{0} d_{1}}, \quad \mathrm{Sr}=\frac{\rho D K_{T}\left(T_{1}-T_{0}\right)}{\eta_{0} T_{m}\left(C_{1}-C_{0}\right)}, \quad \operatorname{Pr}=\frac{\xi \eta_{0}}{k}, \\
& \mathrm{Sc}=\frac{\eta_{0}}{\rho D}, \quad \mathrm{Er}=\frac{c^{2}}{\xi\left(T_{1}-T_{0}\right)}, \quad \nu=\frac{C-C_{0}}{C_{1}-C_{0}}, \\
& \Phi=\frac{d_{1}^{2}}{\eta_{0} c^{2}} \bar{\Phi}, \quad \eta=\frac{T-T_{0}}{T_{1}-T_{0}}, \quad M^{2}=\frac{\sigma B_{0}^{2} d_{1}^{2}}{\eta_{0}} .
\end{aligned}
$$

The Eq. (4) is identically satisfied and Eqs. (5) to (8) in terms of stream function can be expressed as follows:

$$
\begin{aligned}
\delta \operatorname{Re} & {\left[\left(\frac{\partial \psi}{\partial y} \frac{\partial}{\partial x}-\frac{\partial \psi}{\partial x} \frac{\partial}{\partial y}\right) \frac{\partial \psi}{\partial y}\right]=-\frac{\partial p}{\partial x}+\delta \frac{\partial S_{x x}}{\partial x} } \\
+ & \frac{\partial S_{x y}}{\partial y}+\frac{\operatorname{Re}}{F r} \sin \omega-M^{2} \cos \gamma \\
& {\left[\left(\frac{\partial \psi}{\partial y}+1\right) \cos \gamma+\delta \frac{\partial \psi}{\partial x} \sin \gamma\right], } \\
-\delta^{3} \operatorname{Re} & {\left[\left(\frac{\partial \psi}{\partial y} \frac{\partial}{\partial x}-\frac{\partial \psi}{\partial x} \frac{\partial}{\partial y}\right) \frac{\partial \psi}{\partial x}\right]=-\frac{\partial p}{\partial y} } \\
+ & \delta^{2} \frac{\partial S_{x y}}{\partial x}+\delta \frac{\partial S_{y y}}{\partial y}-\delta \frac{\operatorname{Re}}{F r} \cos \omega+M^{2} \delta \cos \gamma \\
& {\left[\left(\frac{\partial \psi}{\partial y}+1\right) \cos \gamma+\delta \frac{\partial \psi}{\partial x} \sin \gamma\right] . }
\end{aligned}
$$

The resulting heat and thermos-diffusion equations become:

$$
\begin{gathered}
\delta \operatorname{Re}\left[\frac{\partial \psi}{\partial y} \frac{\partial \eta}{\partial x}-\frac{\partial \psi}{\partial x} \frac{\partial \eta}{\partial y}\right]=\frac{1}{\operatorname{Pr}}\left(\delta^{2} \frac{\partial^{2} \eta}{\partial x^{2}}+\frac{\partial^{2} \eta}{\partial y^{2}}\right)+\operatorname{Er} \Phi \\
\delta \operatorname{Re}\left[\frac{\partial \psi}{\partial y} \frac{\partial \nu}{\partial x}-\frac{\partial \psi}{\partial x} \frac{\partial \nu}{\partial y}\right]=\frac{1}{\operatorname{Sc}}\left(\delta^{2} \frac{\partial^{2} \nu}{\partial x^{2}}+\frac{\partial^{2} \nu}{\partial y^{2}}\right) \\
+\operatorname{Sr}\left(\delta^{2} \frac{\partial^{2} \eta}{\partial x^{2}}+\frac{\partial^{2} \eta}{\partial y^{2}}\right)
\end{gathered}
$$

Here, bars have been omitted for simplicity.

Solving Eqs. (15) and (16), simultaneously, we get the following vorticity transport equation:

$$
\begin{aligned}
& \delta \operatorname{Re}\left[\left(\frac{\partial \psi}{\partial y} \frac{\partial}{\partial x}-\frac{\partial \psi}{\partial x} \frac{\partial}{\partial y}\right) \nabla^{2} \psi\right]=\left(\frac{\partial^{2}}{\partial y^{2}}-\delta^{2} \frac{\partial^{2}}{\partial x^{2}}\right) S_{x y} \\
& \quad+\delta \frac{\partial^{2}}{\partial x \partial y}\left(S_{x x}-S_{y y}\right)-M^{2} \frac{\partial^{2} \psi}{\partial y^{2}}\left[\left(\frac{\partial \psi}{\partial y}+1\right) \cos \gamma\right. \\
& \left.\quad+\delta \frac{\partial \psi}{\partial x}\right]
\end{aligned}
$$

where:

$$
\nabla^{2}=\delta^{2} \frac{\partial^{2}}{\partial x^{2}}+\frac{\partial^{2}}{\partial y^{2}}
$$

The components of the extra-stress tensor are:

$$
\begin{aligned}
& S_{x x}=4 \delta \psi_{x y}-4 \kappa\left[\delta^{2} \psi_{y} \psi_{x x y}-\delta^{2} \psi_{x} \psi_{x y y}-2 \delta \psi_{x y}^{2}\right. \\
& \left.+\delta^{2} \psi_{x x} \psi_{y y}-\delta^{4} \psi_{x x}^{2}\right] \\
& S_{x y}=2\left(\psi_{y y}-\delta^{2} \psi_{x x}\right)-2 \kappa\left[\delta \psi_{y} \psi_{x y y}-\delta^{3} \psi_{y} \psi_{x x x}\right. \\
& -\delta \psi_{x} \psi_{y y y}+\delta^{3} \psi_{x} \psi_{x x y}-3 \delta \psi_{x y} \psi_{x y} \\
& \left.+\delta \psi_{y y} \psi_{x y}+\delta^{3} \psi_{x y} \psi_{x x}-\delta^{3} \psi_{x x} \psi_{x y}\right] \\
& S_{y y}=-4 \delta \psi_{x y}-4 \kappa\left[\delta^{2} \psi_{y} \psi_{x x y}+\delta^{2} \psi_{x} \psi_{x y y}\right. \\
& \left.-\psi_{y y}^{2}+\delta^{2} \psi_{x x} \psi_{y y}-2 \delta^{2} \psi_{x y}^{2}\right] \\
& \Phi=\left[4 \delta^{2} \psi_{x y}^{2}-4 \kappa \delta^{2} \psi_{x y} \psi_{x x y}+4 \kappa \delta^{3} \psi_{x} \psi_{x y} \psi_{x y y}\right. \\
& +8 \kappa \delta^{3} \psi_{x y}^{3}-12 \kappa \delta^{3} \psi_{x y} \psi_{y y} \psi_{x x}+6 \kappa \delta^{5} \psi_{x y} \psi_{x x}^{2} \\
& +2 \psi_{y y}^{2}-4 \delta^{2} \psi_{y y} \psi_{x x}-2 \kappa \delta \psi_{y} \psi_{y y} \psi_{x y y} \\
& +2 \kappa \delta^{3} \psi_{y} \psi_{y y} \psi_{x x x}+2 \kappa \delta \psi_{x} \psi_{y y} \psi_{y y y} \\
& -2 \kappa \delta^{3} \psi_{x} \psi_{y y} \psi_{x x y}+6 \kappa \delta \psi_{x y} \psi_{y y}^{2}-6 \kappa \delta \psi_{y y} \psi_{x y} \\
& +2 \kappa \delta^{3} \psi_{y y} \psi_{x y} \psi_{x x}+2 \delta^{4} \psi_{x x}^{2}+2 \kappa \delta^{3} \psi_{y} \psi_{x x} \psi_{x y y} \\
& -2 \kappa \delta^{5} \psi_{y} \psi_{x x} \psi_{x x x}-2 \kappa \delta^{3} \psi_{x} \psi_{x x} \psi_{y y y} \\
& +2 \kappa \delta^{5} \psi_{x} \psi_{x x} \psi_{x x y}+6 \kappa \delta^{4} \psi_{x x} \psi_{x y}+4 \delta^{2} \psi_{x y}^{2} \\
& \left.-4 \kappa \delta^{3} \psi_{y} \psi_{x y} \psi_{x x y}+2 \kappa \delta^{3} \psi_{x} \psi_{x y} \psi_{x y y}-8 \kappa \delta^{3} \psi_{x y}^{3}\right]
\end{aligned}
$$

\section{Boundary conditions}

In wave frame, the equations of boundary conditions for dimensionless stream function are:

$$
\begin{aligned}
& \psi=\frac{F}{2}, \frac{\partial \psi}{\partial y}+\beta S_{x y}=-1, \quad \eta=0, \quad v=0 \text { at } y=h_{1}(x), \\
& \psi=-\frac{F}{2}, \quad \frac{\partial \psi}{\partial y}-\beta S_{x y}=-1, \quad \eta=1, \quad \nu=1 \text { at } y=h_{2}(x) .
\end{aligned}
$$

The dimensionless flux $F$ is defined by the following 
relation [14]:

$$
F=\int_{h_{2}(x)}^{h_{1}(x)} \frac{\partial \psi}{\partial y} d y .
$$

In the laboratory frame, dimensionless average flux is obtained as:

$$
\theta=F+1+d
$$

\section{Methodology}

The perturbation method for small wave number $\delta(\delta<<1)$, which is appropriate in the study of peristalic flow in tubes or channels, is used for solving the problem. Thus, we expand the flow quantities as:

$$
\left.\begin{array}{l}
\psi=\psi_{0}+\delta \psi_{1}+O\left(\delta^{2}\right) \\
\eta=\eta_{0}+\delta \eta_{1}+O\left(\delta^{2}\right) \\
\nu=\nu_{0}+\delta \nu_{1}+O\left(\delta^{2}\right) \\
S=S_{0}+\delta S_{1}+O\left(\delta^{2}\right. \\
Z=Z_{0}+\delta Z_{1}+O\left(\delta^{2}\right)
\end{array}\right\}
$$

Substituting Eq. (28) into Eqs. (17) to (19) along with boundary conditions given in Eqs. (24) and (25), and collecting the like terms of we obtain the following zeroth- and first-order problems.

\subsection{Zeroth-order system}

Collecting the coefficients of like terms of $O(\delta)$, we obtain:

$$
\begin{aligned}
& \frac{\partial^{2} S_{0 x y}}{\partial y^{2}}=M^{2} \cos ^{2} \gamma \frac{\partial^{2} \psi_{0}}{\partial y^{2}}, \\
& \frac{1}{\operatorname{Pr}} \frac{\partial^{2} \eta_{0}}{\partial y^{2}}+\operatorname{Er} \Phi_{0}=0 \\
& \frac{1}{\operatorname{Sc}} \frac{\partial^{2} \nu_{0}}{\partial y^{2}}+\operatorname{Sr} \frac{\partial^{2} \eta_{0}}{\partial y^{2}}=0 \\
& S_{0 x x}=0, \quad S_{0 x y}=2 \frac{\partial^{2} \psi_{0}}{\partial y^{2}}, \\
& S_{0 y y}=4 \kappa\left(\frac{\partial^{2} \psi_{0}}{\partial y^{2}}\right)^{2}, \Phi_{0}=2\left(\frac{\partial^{2} \psi_{0}}{\partial y^{2}}\right)^{2} .
\end{aligned}
$$

The corresponding boundary conditions are:

$$
\begin{aligned}
\psi_{0} & =\frac{F_{0}}{2}, \quad \frac{\partial \psi_{0}}{\partial y}+\beta S_{0 x y}=-1, \quad \eta_{0}=0, \\
\nu_{0} & =0 \text { at } y=h_{1}(x), \\
\psi_{0} & =-\frac{F_{0}}{2}, \quad \frac{\partial \psi_{0}}{\partial y}-\beta S_{0 x y}=-1, \quad \eta_{0}=1, \\
\nu_{0} & =1 \text { at } y=h_{2}(x) .
\end{aligned}
$$

The heat coefficient at upper and lower walls can be calculated by the relations:

$$
Z_{0 h_{1}}=\frac{\partial h_{1}}{\partial x} \frac{\partial \eta_{0}}{\partial y}, \quad Z_{0 h_{2}}=\frac{\partial h_{2}}{\partial x} \frac{\partial \eta_{0}}{\partial y} .
$$

\subsection{First-order system}

The coefficients of $\delta$ yield the following problems:

$$
\begin{aligned}
& \frac{\partial^{2} S_{1 x y}}{\partial y^{2}}+\frac{\partial^{2} S_{0 x x}}{\partial x \partial y}=\frac{\partial^{2} S_{0 y y}}{\partial x \partial y} \\
& +\operatorname{Re}\left[\frac{\partial \psi_{0}}{\partial y} \frac{\partial^{3} \psi_{0}}{\partial x \partial y^{2}}-\frac{\partial \psi_{0}}{\partial x} \frac{\partial^{3} \psi_{0}}{\partial y^{3}}\right] \\
& +M^{2}\left[\cos ^{2} \gamma\left(\frac{\partial^{2} \psi_{0}}{\partial x \partial y}+\frac{\partial^{2} \psi_{1}}{\partial y^{2}}\right)\right. \\
& \left.+\sin \gamma \cos \gamma \frac{\partial^{2} \psi_{0}}{\partial x \partial y}\right] \\
& \frac{1}{\operatorname{Pr}} \frac{\partial^{2} \eta_{1}}{\partial y^{2}}+\operatorname{Er} \Phi_{1}=\operatorname{Re}\left[\frac{\partial \psi_{0}}{\partial y} \frac{\partial \eta_{0}}{\partial x}-\frac{\partial \psi_{0}}{\partial x} \frac{\partial \eta_{0}}{\partial y}\right] \\
& \frac{1}{\operatorname{Sc}} \frac{\partial_{1}^{2} \nu}{\partial y^{2}}+\operatorname{Sr} \frac{\partial^{2} \eta_{1}}{\partial y^{2}}=\operatorname{Re}\left[\frac{\partial \psi_{0}}{\partial y} \frac{\partial \nu_{0}}{\partial x}-\frac{\partial \psi_{0}}{\partial x} \frac{\partial \nu_{0}}{\partial y}\right] \\
& S_{1 x x}=4 \frac{\partial^{2} \psi_{0}}{\partial x \partial y}+8 \kappa\left(\frac{\partial^{2} \psi_{0}}{\partial x \partial y}\right)^{2}, \\
& S_{1 x y}=2 \frac{\partial^{2} \psi_{1}}{\partial y^{2}}-2 \kappa \frac{\partial \psi_{0}}{\partial y} \frac{\partial^{3} \psi_{0}}{\partial x \partial y^{2}}+2 \kappa \frac{\partial \psi_{0}}{\partial x} \frac{\partial^{3} \psi_{0}}{\partial y^{3}} \\
& +4 \kappa \frac{\partial^{2} \psi_{0}}{\partial x \partial y}+\frac{\partial^{2} \psi_{0}}{\partial y^{2}} \\
& S_{1 y y}=8 \kappa \frac{\partial^{2} \psi_{0}}{\partial y^{2}} \frac{\partial^{2} \psi_{1}}{\partial y^{2}}-4 \frac{\partial^{2} \psi_{0}}{\partial x \partial y}, \\
& \Phi_{1}=4 \frac{\partial^{2} \psi_{0}}{\partial y^{2}}\left(\frac{\partial^{2} \psi_{1}}{\partial y^{2}}\right)^{2} \\
& +6 \kappa\left[\frac{\partial^{2} \psi_{0}}{\partial x \partial y}\left(\frac{\partial^{2} \psi_{0}}{\partial y^{2}}\right)^{2}-\frac{\partial^{2} \psi_{0}}{\partial x \partial y} \frac{\partial^{2} \psi_{0}}{\partial y^{2}}\right] \\
& +2 \kappa\left[\frac{\partial \psi_{0}}{\partial x} \frac{\partial^{2} \psi_{0}}{\partial y^{2}} \frac{\partial^{3} \psi_{0}}{\partial y^{3}}-\frac{\partial \psi_{0}}{\partial y} \frac{\partial^{2} \psi_{0}}{\partial y^{2}} \frac{\partial^{3} \psi_{0}}{\partial x \partial y^{2}}\right] .
\end{aligned}
$$

The corresponding boundary conditions are:

$$
\begin{aligned}
& \psi_{1}=\frac{F_{1}}{2}, \quad \frac{\partial \psi_{1}}{\partial y}+\beta S_{1 x y}=0, \quad \eta_{1}=0 \\
& \nu_{1}=0, \quad \text { at } \quad y=h_{1}(x), \\
& \psi_{1}=-\frac{F_{1}}{2}, \quad \frac{\partial \psi_{1}}{\partial y}-\beta S_{1 x y}=0, \quad \eta_{1}=0 \\
& \nu_{1}=0 \quad \text { at } y=h_{2}(x) .
\end{aligned}
$$


The heat coefficients at upper and lower walls are defined by the relations:

$$
Z_{1 h_{1}}=\frac{\partial h_{1}}{\partial x} \frac{\partial \eta_{1}}{\partial y}, \quad Z_{1 h_{2}}=\frac{\partial h_{2}}{\partial x} \frac{\partial \eta_{1}}{\partial y}
$$

\section{Solution of the problem}

\subsection{Zeroth-order solution}

The solutions of Eqs. (29) to (36) along with the heat coefficient at upper and lower walls are given by:

$$
\begin{aligned}
& \psi_{0}=A_{1} y+A_{2}+A_{3} \cosh \alpha y+A_{4} \sinh \alpha y, \\
& S_{0 x y}=2 \alpha^{2}\left(A_{3} \cosh \alpha y+A_{4} \sinh \alpha y\right), \\
& \eta_{0}=A_{5}+A_{6} y+A_{7} y^{2}+A_{8} \cosh 2 \alpha y+A_{9} \sinh 2 \alpha y,
\end{aligned}
$$

$\nu_{0}=A_{10}+A_{11} y+A_{12} y^{2}+A_{13} \cosh 2 \alpha y+A_{14} \sinh 2 \alpha y$,

$$
\begin{aligned}
Z_{0 h_{1}} & =a \cos x\left(A_{6}+2 A_{7} y+2 \alpha A_{8} \sinh \alpha y\right. \\
& \left.+2 \alpha A_{9} \cosh 2 \alpha y\right)
\end{aligned}
$$

$$
\begin{aligned}
Z_{0 h_{2}}= & -b \cos (x+\phi)\left(A_{6}+2 A_{7} y+2 \alpha A_{8} \sinh 2 \alpha y\right. \\
& \left.+2 \alpha A_{9} \cosh 2 \alpha y\right) .
\end{aligned}
$$

\subsection{First-order solution}

Upon making use of zeroth-order solution in the firstorder system and then solving the resulting problems, one arrives at:

$$
\begin{aligned}
\psi_{1}= & B_{1}+B_{2} y+\cosh \alpha y\left(B_{4}+B_{5} y+B_{6} y^{2}\right) \\
& +B_{3} y^{2}+\sinh \alpha y\left(B_{7}+B_{8} y+B_{9} y^{2}\right) \\
S_{1 x y}= & D_{10}+\cosh \alpha y\left(B_{11}+B_{12} y+B_{13} y^{2}\right) \\
& +B_{17} \cosh 2 \alpha y+\sinh \alpha y\left(B_{14}+B_{15} y+B_{16} y^{2}\right) \\
& +B_{18} \sinh 2 \alpha y . \\
\eta_{1}= & B_{19}+B_{20} y+B_{22} y^{3} \sinh \alpha y\left(B_{24}+B_{25} y\right. \\
+ & \left.B_{26} y^{2}\right)+\cosh \alpha y\left(B_{27}+B_{28} y+B_{29} y^{2}\right) \\
+ & B_{36} \sinh 3 \alpha y+\sinh 2 \alpha y\left(B_{30}+B_{31} y\right. \\
+ & \left.B_{32} y^{2}\right)+B_{23} y^{4}+B_{21} y^{2}+\cosh 2 \alpha y\left(B_{33}\right. \\
+ & \left.B_{34} y+B_{35} y^{2}\right)+B_{37} \cosh 3 \alpha y
\end{aligned}
$$

$$
\begin{aligned}
\nu_{1}= & B_{38}+B_{39} y+B_{40} y^{2}+\sinh \alpha y\left(B_{43}+B_{44} y\right. \\
& \left.+B_{45} y^{2}\right)+B_{41} y^{3}+B_{42} y^{4}+\cosh \alpha y\left(B_{46}\right. \\
& \left.+B_{47} y+B_{48} y^{2}\right)+\sinh 2 \alpha y\left(B_{49}+B_{50} y\right. \\
& \left.+B_{51} y^{2}\right)+B_{55} \sinh 3 \alpha y+\cosh 2 \alpha y\left(B_{52}\right. \\
& \left.+B_{53} y+B_{54} y^{2}\right)+B_{56} \cosh 3 \alpha y .
\end{aligned}
$$

Heat transfer coefficient is:

$$
\begin{aligned}
& Z_{1 h_{1}}=a \cos x\left(\operatorname { c o s h } \alpha y \left(B_{28}+\alpha B_{24}+\left(\alpha B_{25}\right.\right.\right. \\
& \left.\left.+2 B_{29}\right) y+\alpha B_{26} y^{2}\right)+\sinh \alpha y\left(B_{25}\right. \\
& \left.+\alpha B_{27}+\left(\alpha B_{28}+2 B_{26}\right) y+2 \alpha B_{29} y^{2}\right) \\
& +2 B_{21} y+\cosh 2 \alpha y\left(B_{34}+2 \alpha B_{30}+\left(2 \alpha B_{31}\right.\right. \\
& \left.\left.+2 B_{35}\right) y+2 \alpha B_{32} y^{2}\right)+B_{20}+\sinh 2 \alpha y\left(B_{31}\right. \\
& \left.+2 \alpha L_{33}+\left(2 \alpha B_{34}+2 B_{32}\right) y+2 \alpha B_{35} y^{2}\right) \\
& +3 \alpha B_{36} \sinh 3 \alpha y+3 \alpha B_{37} \cosh 3 \alpha y \\
& \left.+3 B_{22} y^{2}+4 B_{23} y^{3}\right), \\
& Z_{1 h_{2}}=-b \cos (x+\phi)\left(\operatorname { c o s h } \alpha y \left(B_{28}+\alpha B_{24}+\left(\alpha B_{25}\right.\right.\right. \\
& \left.\left.+2 B_{29}\right) y+\alpha B_{26} y^{2}\right)+\sinh \alpha y\left(B_{25}+\alpha B_{27}\right. \\
& \left.+\left(\alpha B_{28}+2 B_{26}\right) y+2 \alpha B_{29} y^{2}\right)+3 B_{22} y^{2} \\
& +\cosh 2 \alpha y\left(B_{34}+2 \alpha B_{30}+\left(2 \alpha B_{31}+2 B_{35}\right) y\right. \\
& \left.+2 \alpha B_{32} y^{2}\right)+4 B_{23} y^{3}+\sinh 2 \alpha y\left(B_{31}\right. \\
& \left.+2 \alpha L_{33}+\left(2 \alpha B_{34}+2 B_{32}\right) y+2 \alpha B_{35} y^{2}\right) \\
& +B_{20}+2 B_{21} y+3 \alpha B_{36} \sinh 3 \alpha y \\
& \left.+3 \alpha B_{37} \cosh 3 \alpha y\right) .
\end{aligned}
$$

The values appearing in Eqs. (47) to (58) are given in the Appendix.

\section{Results and discussion}

In this section, the effects of physical parameters such as viscoelastic parameter, $\kappa$; MHD parameter, $M$; slip parameter, $\beta$; Eckert number, ER; Prandtl number, Pr; Schmidt number, Sc; and Soret number, Sr, have been displayed in Figures 1 to 11.

Figures 1 to 3 are prepared to discuss the variations of $\kappa, M$, and $\beta$ on shear stress $S_{x y}$. Figure 1 depicts that, by increasing $K$, the absolute value of 


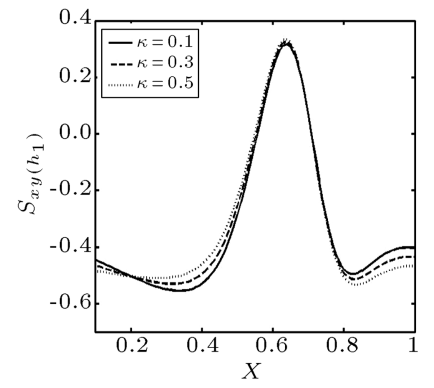

(a)

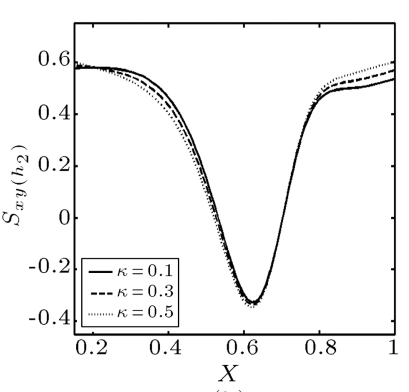

(b)
Figure 1. Variations of $\kappa$ on (a) $S_{x y\left(h_{1}\right)}$ and (b) $S_{x y\left(h_{2}\right)}$.

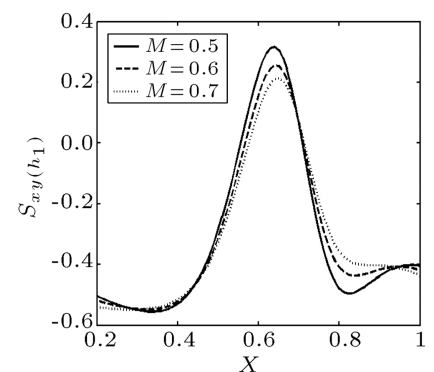

(a)

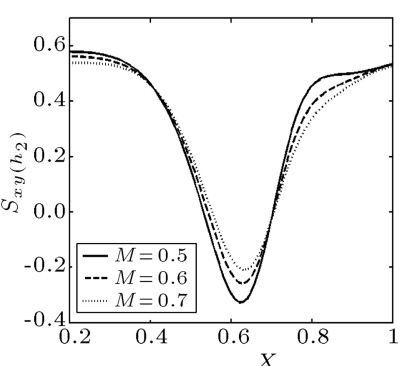

(b)
Figure 2. Variations of $M$ on (a) $S_{x y\left(h_{1}\right)}$ and (b) $S_{x y\left(h_{2}\right)}$.

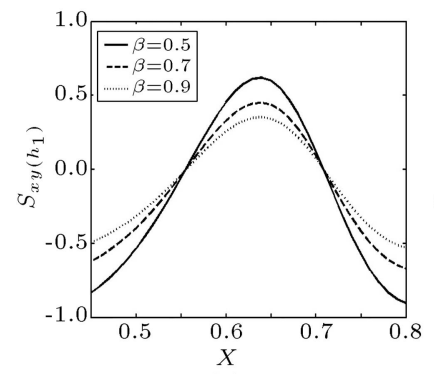

(a)

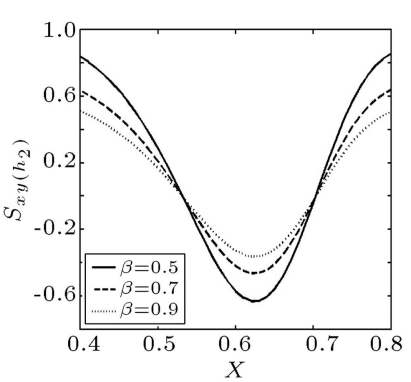

(b)
Figure 3. Variations of $\beta$ on (a) $S_{x y\left(h_{1}\right)}$ and (b) $S_{x y\left(h_{2}\right)}$.

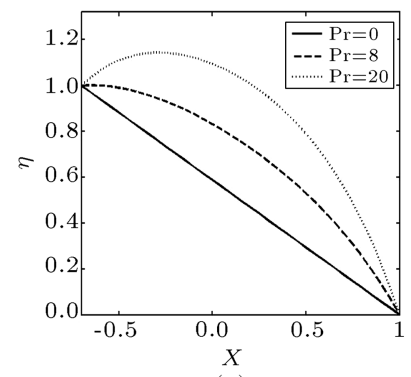

(a)

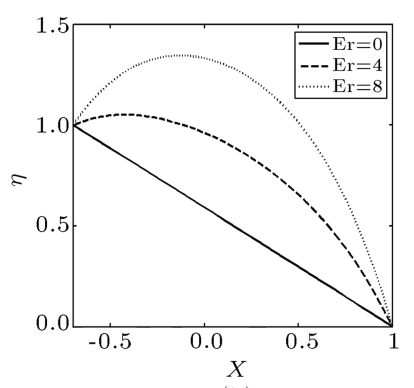

(b)
Figure 4. Variations of (a) Pr and (b) Er on $\eta$.

shear stress at the upper wall $S_{x y\left(h_{1}\right)}$ and lower wall $S_{x y\left(h_{2}\right)}$, respectively, decreases and increases in the first half of the wave and then increases and decreases in the second half of the wave as increasing $\kappa$. Figure 2 shows that there is decrease in the absolute value of shear stress when $M$ increases. From Figure 3, it is found that with increasing the value of $\beta$, the absolute value of shear stress decreases.

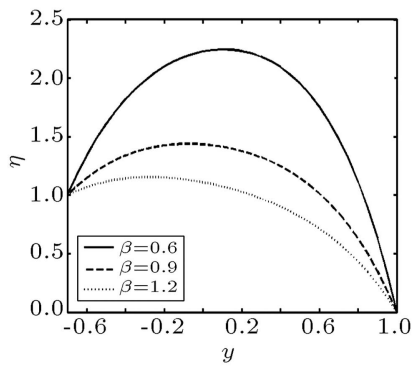

(a)

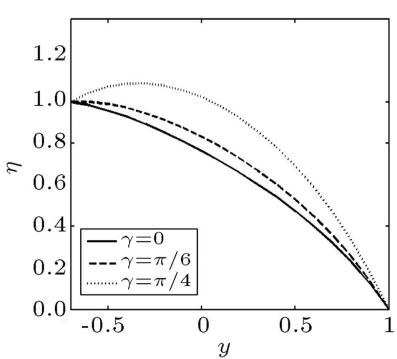

(b)
Figure 5. Variations of (a) $\beta$ and (b) $\gamma$ on $\eta$.

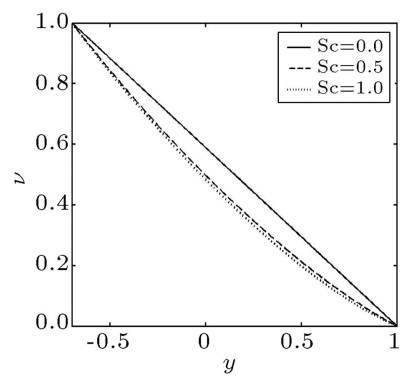

(a)

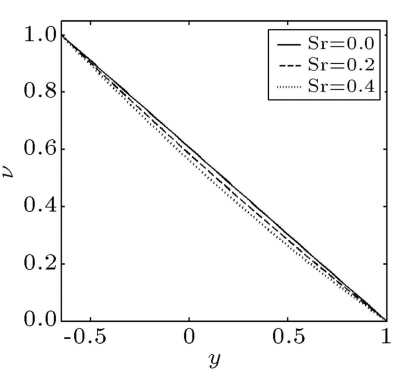

(b)
Figure 6. Variations of (a) Sc and (b) Sr on $\nu$.

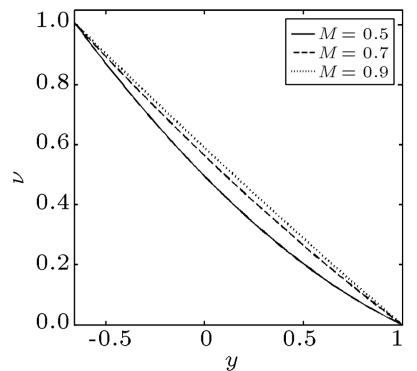

(a)

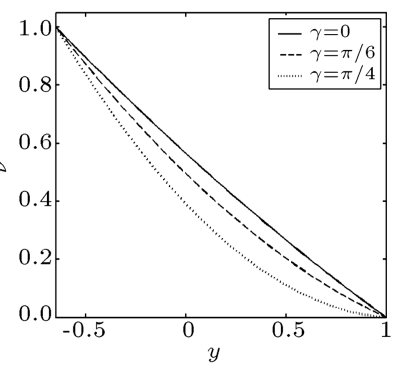

(b)
Figure 7. Variation of (a) $M$ and (b) on $\nu$.

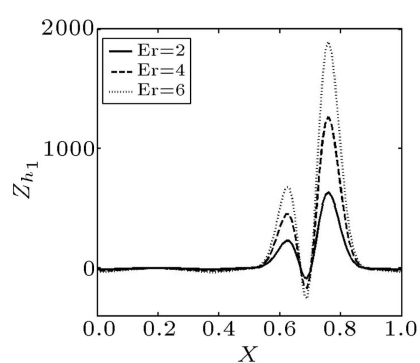

(a)

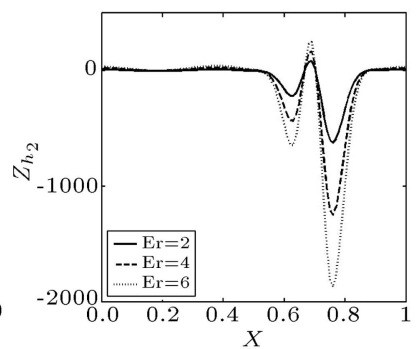

(b)
Figure 8. Variations of Er on heat transfer coefficients: (a) $Z_{h_{1}}$ at upper wall; and (b) $Z_{h_{2}}$ at lower wall.

Figures 4 and 5 show the variations of Prandtl number, Pr, Eckert number, Er, and slip parameter, $\beta$, at inclination of magnetic field $\gamma$ on temperature $\eta$ From Figure 4, it is clear that by increasing Pr and Er, temperature $\eta$ increases. Figure 5 (a) illustrates the decrease in temperature $\eta$ with increase in $\beta$, whereas from Figure $5(\mathrm{~b})$, it is observed that temperature $\eta$ increases by increasing $\gamma$. 


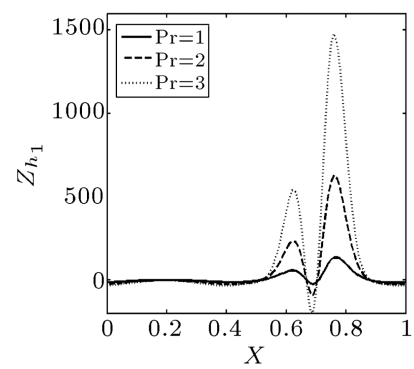

(a)

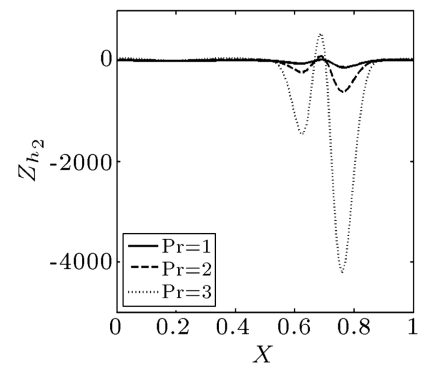

(b)
Figure 9. Variations of $\operatorname{Pr}$ on heat transfer coefficients: (a) $Z_{h_{1}}$; and (b) $Z_{h_{2}}$.

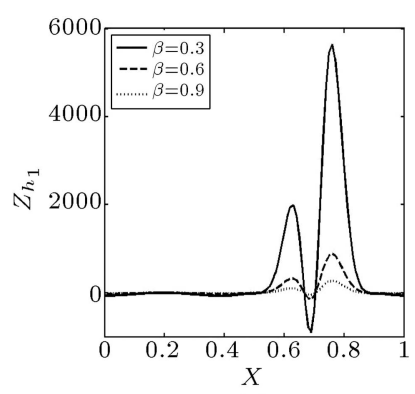

(a)

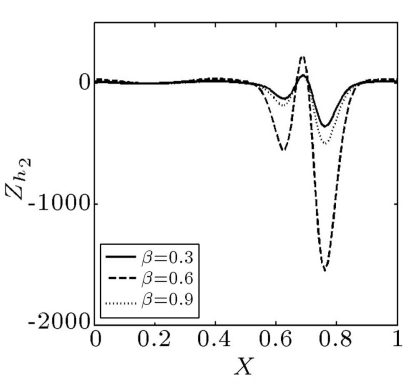

(b)
Figure 10. Variations of $\beta$ on heat transfer coefficients: (a) $Z_{h_{1}}$ at upper wall; and (b) $Z_{h 2}$ at lower wall.

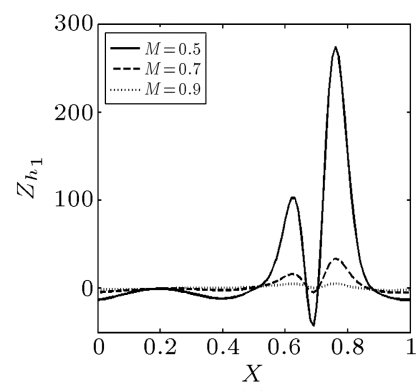

(a)

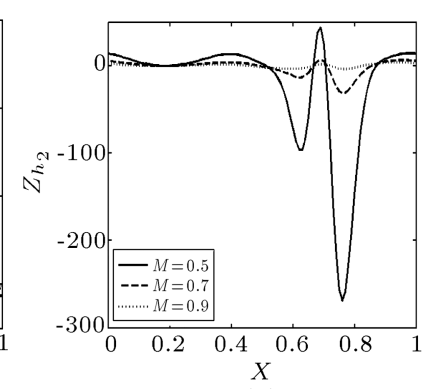

(b)
Figure 11. Variations of $M$ on heat transfer coefficients: (a) $Z_{h_{1}}$ at upper wall; and (b) $Z_{h_{2}}$ at lower wall.

These are not unexpected results as, based on the definition, the Prandtl number is a ratio of momentum diffusion to thermal diffusion; consequently, with increase in Pr, thermal diffusion decreases and, thus, thermal boundary layer becomes thinner. In other words, one can say that large values of Prandtl number result in thinning of thermal boundary layer. As a matter of fact, the increase of Prandtl number slows the rate of thermal diffusion. However, in the presence of viscous dissipation, the effect of increasing the values of Prandtl number is to increase temperature distribution, whereas in the absence of viscous dissipation, the effect of increasing Prandtl number, $\mathrm{Pr}$, is to increase the heat transfer rate, significantly, which are absolutely in accordance with the physical expectation. In addition, the presented work is in contrast to the effects of other parameters that show the same tendency as that already reported in existing literature $[15,16]$.

Figures 6 and 7 indicate the behavior of parameters on $\nu$. The effects of $\mathrm{SC}$ and $\mathrm{Sr}$ on $\nu$ are indicated through Figure 6 . In this figure, $\nu$ decreases by increasing Sc and Sr. Figure 7(a) illustrates the effect of $M$ on $V$. It is clearly observed that $\nu$ increases by increasing $M$. Figure 7 (b) represents the influence of $\gamma$ on $\nu$. It is seen that $\nu$ decreases by increasing the value of $\gamma$.

Figures 8 to 11 are plotted to study the effects of heat transfer coefficients $Z_{h_{1}}$ and $Z_{h_{2}}$ for different values of $\operatorname{Er}, \operatorname{Pr}, \beta$, and $M$. Heat transfer coefficient, $Z$, has oscillatory behavior, which is due to the propagation of peristaltic waves along the walls of the channel. Figures 8 and 9 represent that the absolute value of heat transfer coefficient increases by increasing the values of Er and Pr. Figure 10 depicts that the absolute value of heat transfer coefficient decreases at the upper wall, but increases at the lower wall of the channel by increasing $\beta$. Figure 11 indicates that the absolute value of heat transfer coefficient $Z$ decreases when $M$ increases.

\section{Conclusion}

In this study, the combined effect of slip condition, heat transfer, and chemical reactions on the peristaltic flow of Walter's B fluid in an inclined asymmetric channel has been investigated. The system of partial differential equations is first written in terms of ordinary differential equations by using adequate similarity transformations and then solved analytically through perturbation method. In particular, analytic solutions have been developed for shear stress $S_{x, y}$, temperature $\eta$, concentration $\nu$, and heat transfer coefficient $Z$. The results are discussed through graphs. The main observations in this study are:

- The absolute value of shear stress, $S_{x y}$, decreases as $M$ increases at the upper and lower walls;

- The temperature $\eta$ decreases with an increase in $\beta$, while with increase in $\operatorname{Pr}, \operatorname{Er}$, and $\gamma$, the temperature $\eta$ increases;

- The absolute value of heat transfer coefficient, $Z$, decreases by increasing $M$ and $\beta$, but the reverse behavior is noted by increasing the values of $\mathrm{Pr}, \mathrm{Er}$, and $\gamma$ at the upper wall of the channel;

- The absolute value of heat transfer coefficient, $Z$, decreases by increasing $M$ at the lower wall, whereas its behavior is quite opposite for increasing values of Pr, Er, $\beta$, and $\gamma$;

- The concentration $\eta$ increases with the increase of $M$; however, with increase in $\mathrm{Sr}, \mathrm{Sc}$, and $\gamma$, the concentration $\eta$ decreases. 


\section{Nomenclature}

\section{References}

1. Latham, T. W. "Fluid motion in peristaltic pump", MS thesis, Massachusetts Institute of Technology (1996).

2. Kothandapani, M. and Srinivas, S. "Peristaltic transport in an asymmetric channel with heat transfer-a note", Int. Comm. Heat and Mass Transfer, 35, pp. 514-522 (2008).

3. Nadeem, S. and N. S. Akbar, "Influence of heat transfer on a peristaltic transport of Herschel Bulkley fluid in a non-uniform inclined tube", Commun. Nonlinear Sci. Numer. Simulat., 14, pp. 4100-4113 (2009).

4. Mansur, S. Ishak, A. and Pop, I. "Flow and heat transfer of nanofluid past stretching/shrinking sheet with partial slip boundary conditions", Applied Mathematics and Mechanics, 35(11), pp. 1401-1410 (2014).

5. Kefayati, G. H.R. Gorji-Bandpy, M. Sajjadi, H. and Ganji, D. D., "Lattice Boltzmann simulation of MHD mixed convection in a lid-driven square cavity with linearly heated wall", Scientia Iranica, 19(4), pp. 10531065 (2012).

6. Beard, D. W. and Walters, K. "Elastico-viscous boundary layer flows", Cambridge Philos. Soc., 60, pp. 667674 (1964).

7. Rajagopal, K. R. Na, T. Y. and Gupta, A. S. "Flow of a viscoelastic fluid over a stretching sheet", Rheo. Acta, 23, pp. 213-21 (1984).
8. Malik, M.Y. Hussain, A. and Nadeem, S. "Boundary layer flow of an Eyring-Powell model fluid due to a stretching cylinder with variable viscosity", Scientia Iranica, 20(2), pp. 313-321 (2013).

9. Walters, K. "Second-Order Effects in Elasticity, Plasticity and Fluid Dynamics", pp. 519-521, Pergamon, Oxford, UK (1964).

10. Joneidi, A. A. Domairry, G. and Babaelahi, M. "Homotopy analysis method to Walter's B fluid in a vertical channel with porous wall", Meccanica, 45, pp. 857-868 (2010).

11. Nandeppanavar, M. M. Abel, M. S. and Tawade, J. "Heat transfer in a Walters liquid B fluid over an impermeable stretching sheet with non-uniform heat source/sink and elastic deformation", Commun. NonLinear Sci. Numer. Simul., 15, pp. 1791-1802 (2010).

12. Hayat, T. Asad, S. Mustafa M. and Alsulami, H. "Heat transfer analysis in the flow of Walters' B fluid with a convective boundary condition", Chin. Phys. B, 23(8), pp. 084701-7 (2014).

13. Baris, S. "Steady Three-Dimensional Flow of a Walter's B' Fluid in a Vertical Channel", Turk. J. Eng. Env. Sci., 26, pp. 385-294 (2002).

14. Shapiro, A. H. Jaffrin, M. Y. and Weinberg, S. L. "Peristaltic pumping with long wavelengths at low Reynolds number", J. Fluid Mech., pp. 799-825 (1969).

15. Rashidi, M. M. Rostami, B. Navid Freidoonimehr, N. and Abbasbandy, S. "Free convective heat and mass transfer for MHD fluid flow over a permeable vertical stretching sheet in the presence of the radiation and buoyancy effects", Ain Shams Engineering Journal,5, pp. 901-912 (2014).

16. Subhas Abel, M. and Mahesha, N. "Heat transfer in MHD viscoelastic fluid flow over a stretching sheet with variable thermal conductivity, non-uniform heat source and radiation", Applied Mathematical Modelling, 32, pp. 1965-1983 (2008).

\section{Appendix}

$$
\begin{aligned}
A_{1}= & \frac{F_{0}}{h_{1}-h_{2}}-\frac{\left(F_{0}+h_{1}-h_{2}\right)\left(C_{6} C_{1}-C_{2} C_{5}\right)}{\left(h_{1}-h_{2}\right) C_{5}}, \\
A_{2}= & -\frac{F_{0}\left(h_{1}+h_{2}\right)}{2\left(h_{1}-h_{2}\right)}-\frac{\left(F_{0}+h_{1}-h_{2}\right) C_{9}}{\left(h_{1}-h_{2}\right) C_{7}} \\
A_{3}= & \frac{\left(F_{0}+h_{1}-h_{2}\right) C_{6}}{C_{5}} \\
A_{4}= & -\frac{\left(F_{0}+h_{1}-h_{2}\right)}{\left(h_{1}-h_{2}\right) C_{7}}, \\
A_{5}= & \frac{1}{\left(h_{2}-h_{1}\right)}\left[-h_{1}+A_{7} h_{2} h_{1}\left(h_{2}-h_{1}\right)\right. \\
& +A_{8}\left(h_{1} \cosh 2 \alpha h_{2}-h_{2} \cosh 2 \alpha h_{1}\right) \\
& \left.+A_{9}\left(h_{1} \sinh 2 \alpha h_{2}-h_{2} \sinh 2 \alpha h_{1}\right)\right]
\end{aligned}
$$




$$
\begin{aligned}
& A_{6}=\frac{1}{\left(h_{2}-h_{1}\right)}\left[1-A_{7}\left(h_{2}^{2}-h_{1}^{2}\right)\right. \\
& -A_{8}\left(\cosh 2 \alpha h_{2}-\cosh 2 \alpha h_{1}\right) \\
& \left.-A_{9}\left(\sinh 2 \alpha h_{2}-\sinh 2 \alpha h_{1}\right)\right] \text {, } \\
& A_{7}=\frac{\operatorname{Er} \operatorname{Pr} \alpha^{4}\left(A_{4}^{2}-A_{3}^{2}\right)}{2}, \\
& A_{8}=\frac{-\operatorname{Er} \operatorname{Pr} \alpha^{2}\left(A_{4}^{2}+A_{3}^{2}\right)}{4}, \\
& A_{9}=\frac{-\operatorname{Er} \operatorname{Pr} \alpha^{2} A_{4} A_{3}}{2}, \\
& A_{10}=\frac{A_{50}-A_{50}\left(1+h_{2}-h_{1}\right)-1}{h_{2}-h_{1}}, \\
& A_{11}=\frac{1-A_{50}+A_{12}}{h_{2}-h_{1}}, \quad A_{12}=-\operatorname{SrSc} A_{7} \\
& A_{13}=-\operatorname{SrSc} A_{8}, \quad A_{14}=-\operatorname{SrSc} A_{9}, \\
& A_{15}=M^{2}\left(\sin \gamma \cos \gamma+\cos ^{2} \gamma\right), \quad A_{16}=A_{1}^{\prime} A_{14}, \\
& A_{17}=\operatorname{Re}\left(A_{1} A_{3}^{\prime} \alpha^{2}-A_{2}^{\prime} A_{4} \alpha^{3}\right)+\kappa\left(-8 A_{1}^{\prime} A_{3} \alpha^{4}\right. \\
& \left.-2 A_{2}^{\prime} A_{4} \alpha^{5}+2 A_{1} A_{3}^{\prime} \alpha^{4}\right)+\alpha A_{4}^{\prime} A_{5}, \\
& A_{18}=\operatorname{Re}\left(A_{1} A_{4}^{\prime} \alpha^{2}-A_{2}^{\prime} A_{3} \alpha^{3}\right)+\kappa\left(-8 A_{1}^{\prime} A_{4} \alpha^{4}\right. \\
& \left.-2 A_{2}^{\prime} A_{3} \alpha^{5}+2 A_{1} A_{4}^{\prime} \alpha^{4}\right)+\alpha A_{3}^{\prime} A_{5}, \\
& A_{19}=-\operatorname{Re} A_{3} A_{1}^{\prime} \alpha^{3}-2 \kappa A_{1}^{\prime} A_{3} \alpha^{5} \text {, } \\
& A_{20}=\operatorname{Re} A_{4} A_{1}^{\prime} \alpha^{3}-2 \kappa A_{1}^{\prime} A_{4} \alpha^{5}, \\
& A_{21}=\frac{A_{49}}{A_{48}}, \\
& A_{22}=\frac{F_{1}}{2}-\frac{A_{49} h_{1}}{A_{48}}-\frac{A_{47} \cosh \alpha h_{1}}{A_{48}} \\
& -\frac{A_{45} \sinh \alpha h_{1}}{A_{46}}-A_{34}, \\
& A_{23}=\frac{A_{47}}{A_{48}}, \quad A_{24}=\frac{A_{45}}{A_{46}}, \\
& A_{25}=\frac{A_{17}\left(h_{2} \sinh \alpha h_{2}-h_{1} \sinh \alpha h_{1}\right)}{4 \alpha^{3}} \\
& +\frac{A_{18}\left(h_{2} \cosh \alpha h_{2}-h_{1} \cosh \alpha h_{1}\right)}{4 \alpha^{3}}-\frac{A_{16}\left(h_{2}^{2}-h_{1}^{2}\right)}{4 \alpha^{2}} \\
& +\frac{\left(\alpha^{2} h_{2}^{2}+5 \alpha h_{2}-4\right)\left(A_{19} \cosh \alpha h_{2}+A_{20} \sinh \alpha h_{2}\right)}{8 \alpha^{5}} \\
& -\frac{\left(\alpha^{2} h_{1}^{2}-5 \alpha h_{1}-4\right)\left(A_{19} \cosh \alpha h_{1}+A_{20} \sinh \alpha h_{1}\right)}{8 \alpha^{5}}, \\
& A_{26}=4 A_{1}^{\prime} A_{3} \alpha^{2}-2 A_{3}^{\prime} A_{1} \alpha^{2}+2 A_{4} A_{2}^{\prime} \alpha^{3}, \\
& A_{27}=2 A_{4} A_{1}^{\prime} \alpha^{3} \text {, } \\
& A_{28}=4 A_{1}^{\prime} A_{4} \alpha^{2}+2 A_{2}^{\prime} A_{3} \alpha^{3}-2 A_{1} A_{4}^{\prime} \alpha^{2}, \\
& A_{29}=2 A_{3} A_{1}^{\prime} \alpha^{3} \text {, } \\
& A_{30}=\frac{1}{2}\left(A_{3} A_{3}^{\prime} \alpha^{3}+A_{4} A_{4}^{\prime} \alpha^{3}\right), \\
& A_{31}=\frac{1}{2}\left(A_{4} A_{3}^{\prime} \alpha^{3}+A_{3} A_{4}^{\prime} \alpha^{3}\right), \\
& A_{32}=-\frac{A_{16} h_{1}}{2 \alpha^{2}}+\sinh \alpha h_{1}\left(\frac{A_{17}}{4 \alpha^{3}}+\frac{A_{18} h_{1}}{4 \alpha^{2}}\right. \\
& \left.+\frac{A_{19} h_{1}^{2}}{8 \alpha^{2}}-\frac{3 A_{20} h_{1}}{8 \alpha^{3}}-\frac{9 A_{19}}{8 \alpha^{4}}\right)+\cosh \alpha h_{1}\left(\frac{A_{18}}{4 \alpha^{3}}\right. \\
& \left.+\frac{A_{17} h_{1}}{4 \alpha^{2}}+\frac{A_{20} h_{1}^{2}}{8 \alpha^{2}}-\frac{3 A_{19} h_{1}}{8 \alpha^{3}}-\frac{9 A_{20}}{8 \alpha^{4}}\right), \\
& A_{33}=\sinh \alpha h_{2}\left(\frac{A_{17}}{4 \alpha^{3}}+\frac{A_{18} h_{2}}{4 \alpha^{2}}+\frac{A_{19} h_{2}^{2}}{8 \alpha^{2}}\right. \\
& \left.-\frac{3 A_{20} h_{2}}{8 \alpha^{3}}-\frac{9 A_{19}}{8 \alpha^{4}}\right)-\frac{A_{16} h_{2}}{2 \alpha^{2}}+\cosh \alpha h_{2} \\
& \left(\frac{A_{18}}{4 \alpha^{3}}+\frac{A_{17} h_{2}}{4 \alpha^{2}}+\frac{A_{20} h_{2}^{2}}{8 \alpha^{2}}-\frac{3 A_{19} h_{2}}{8 \alpha^{3}}-\frac{9 A_{20}}{8 \alpha^{4}}\right), \\
& A_{34}=-\frac{A_{16} h_{1}^{2}}{4 \alpha^{2}}+\sinh \alpha h_{1}\left(\frac{A_{17} h_{1}}{4 \alpha^{3}}-\frac{A_{20} h_{1}^{2}}{8 \alpha^{3}}\right. \\
& \left.-\frac{5 A_{19} h_{1}}{8 \alpha^{4}}-\frac{A_{20}}{2 \alpha^{5}}\right)+\cosh \alpha h_{1}\left(\frac{A_{18} h_{1}}{4 \alpha^{3}}\right. \\
& \left.+\frac{A_{19} h_{1}^{2}}{8 \alpha^{3}}-\frac{3 A_{20} h_{1}}{8 \alpha^{4}}-\frac{A_{19}}{2 \alpha^{5}}\right) \\
& A_{35}=-\frac{A_{16} h 2}{4 \alpha^{2}}+\sinh \alpha h_{2}\left(\frac{A_{17} h_{2}}{4 \alpha^{3}}-\frac{A_{20} h_{2}^{2}}{8 \alpha^{3}}\right. \\
& \left.-\frac{5 A_{19} h_{2}}{8 \alpha^{4}}-\frac{A_{20}}{2 \alpha^{5}}\right)+\cosh \alpha h_{2}\left(\frac{A_{18} h_{2}}{4 \alpha^{3}}\right. \\
& \left.+\frac{A_{19} h_{2}^{2}}{8 \alpha^{3}}-\frac{3 A_{20} h_{2}}{8 \alpha^{4}}-\frac{A_{19}}{2 \alpha^{5}}\right) \\
& A_{36}=\alpha\left(\sinh \alpha h_{2}-\sinh \alpha h_{1}\right) \\
& -2 \alpha^{2} \beta\left(\cosh \alpha h_{2}+\cosh \alpha h_{1}\right), \\
& A_{37}=\alpha\left(\cosh \alpha h_{2}-\cosh \alpha h_{1}\right) \\
& -2 \alpha^{2} \beta\left(\sinh \alpha h_{2}+\sinh \alpha h_{1}\right),
\end{aligned}
$$




$$
\begin{aligned}
& A_{38}=A_{33}-A_{32}-\beta\left(A_{31}+A_{28}\right), \\
& A_{39}=\alpha \sinh \alpha h_{1}+2 \alpha^{2} \beta \cosh \alpha h_{1}, \\
& A_{40}=\alpha \cosh \alpha h_{1}+2 \alpha^{2} \beta \sinh \alpha h_{1}, \\
& B_{22}=\operatorname{Pr}\left[\frac{\operatorname{Re} B_{79}}{6}-\frac{\operatorname{Er}\left(B_{72}-B_{74}\right)}{12}\right] \text {, } \\
& B_{23}=\operatorname{Pr}\left[\frac{\operatorname{Re} B_{80}}{12}-\frac{\operatorname{Er}\left(B_{73}-B_{75}\right)}{24}\right] \text {, } \\
& A_{41}=A_{32}+\beta A_{28}, \\
& A_{42}=\alpha \sinh \alpha h_{2}-2 \alpha^{2} \beta \cosh \alpha h_{2}, \\
& A_{43}=\alpha \cosh \alpha h_{2}-2 \alpha^{2} \beta \sinh \alpha h_{2}, \\
& A_{44}=A_{33}-\beta A_{31}, \\
& A_{45}=-F_{1}-A_{25}-\left(h_{2}-h_{1}\right)\left(\frac{A_{39} A_{38}}{A_{36}}-A_{41}\right) \\
& B_{24}=\operatorname{Pr}\left[\operatorname { R e } \left(\frac{2 B_{85}-B_{93}+B_{94}}{2 \alpha^{2}}-\frac{2 B_{89}}{\alpha^{3}}\right.\right. \\
& \left.\left.+\frac{6 B_{87}}{\alpha^{4}}\right)-\operatorname{Er}\left(\frac{4 B_{16}-3 B_{18}+B_{21}}{4 \alpha^{2}}\right)\right] \\
& B_{25}=\operatorname{Pr} \operatorname{Re}\left(\frac{B_{86}}{\alpha^{2}}-\frac{4 B_{90}}{\alpha^{3}}\right) \text {, } \\
& B_{26}=\operatorname{Pr} \operatorname{Re} \frac{B_{87}}{\alpha^{2}} \\
& +\frac{A_{38}}{A_{36}}\left(\cosh \alpha h_{2}-\cosh \alpha h_{1}\right) \\
& A_{46}=\left(h_{2}-h_{1}\right)\left(\frac{A_{39} A_{37}}{A_{36}}-A_{40}\right)-\frac{A_{37}}{A_{36}} \\
& \left(\cosh \alpha h_{2}-\cosh \alpha h_{1}\right)-\left(\sinh \alpha h_{2}-\sinh \alpha h_{1}\right), \\
& \begin{aligned}
B_{27} & =\operatorname{Pr} \operatorname{Re}\left(\frac{B_{89}}{\alpha^{2}}-\frac{4 B_{87}}{\alpha^{3}}, B_{37}\right. \\
& =\operatorname{Pr}\left[\frac{2 \operatorname{Re}\left(B_{90}+B_{91}\right)-\operatorname{Er}\left(B_{69}+B_{70}\right)}{36 \alpha^{2}}\right],
\end{aligned} \\
& A_{47}=-A_{45} A_{37}-A_{38} A_{46}, \quad A_{48}=A_{36} A_{46}, \\
& A_{49}=A_{39} A_{47}-A_{36}\left(A_{45} A_{40}+A_{41}\right) \text {, } \\
& B_{1}=A_{22}, \quad B_{2}=A_{21}, \\
& B_{3}=-\frac{A_{16}}{2}, \quad B_{4}=A_{23}-\frac{A_{19}}{2 \alpha^{5}}, \\
& B_{5}=\frac{A_{18}}{4 \alpha^{3}}-\frac{5 A_{19}}{8 \alpha^{4}}, B_{6}=\frac{A_{19}}{8 \alpha^{3}}, B_{7}=A_{24}-\frac{A_{20}}{2 \alpha^{5}}, \\
& B_{8}=\frac{A_{17}}{4 \alpha^{3}}-\frac{5 A_{19}}{8 \alpha^{4}}, \quad B_{9}=\frac{A_{20}}{8 \alpha^{3}}, \\
& B_{10}=-\frac{A_{16}}{\alpha^{2}}+\frac{\kappa \alpha^{3}}{2}\left(A_{3} A_{4}^{\prime}-A_{3}^{\prime} A_{4}\right), \\
& B_{11}=\frac{A_{17}}{\alpha^{2}}-\frac{3 A_{19}}{\alpha^{3}}+\kappa A_{26} \\
& B_{12}=\frac{A_{18}}{2 \alpha}-\frac{A_{20}}{4 \alpha^{2}}+\kappa A_{27}, \quad B_{13}=\frac{A_{19}}{4 \alpha}, \\
& B_{14}=\frac{A_{18}}{\alpha^{2}}-\frac{3 A_{20}}{\alpha^{3}}+\kappa A_{28} \\
& B_{15}=\frac{A_{17}}{2 \alpha}-\frac{A_{19}}{4 \alpha^{2}}+\kappa A_{29}, \quad B_{16}=\frac{A_{20}}{4 \alpha}, \\
& B_{17}=\kappa A_{30}, \quad B_{18}=\kappa A_{31}, \\
& B_{19}=\frac{h_{1} B_{21}-h_{2} B_{20}}{h_{2}-h_{1}}, \quad B_{20}=\frac{B_{20}-B_{21}}{h_{2}-h_{1}}, \\
& B_{28}=\operatorname{Pr}\left[\operatorname { R e } \left(\frac{2 B_{88}-B_{91}+B_{92}}{2 \alpha^{2}}-\frac{2 B_{86}}{\alpha^{3}}\right.\right. \\
& \left.\left.+\frac{6 B_{90}}{\alpha^{4}}\right)-\operatorname{Er}\left(\frac{4 B_{67}+3 B_{69}-B_{70}}{4 \alpha^{2}}\right)\right], \\
& B_{29}=\operatorname{Pr} \operatorname{Re} \frac{B_{90}}{\alpha^{2}} \\
& B_{30}=\operatorname{Pr}\left[\operatorname{Re}\left(\frac{B_{83}}{4 \alpha^{2}}-\frac{B_{82}}{4 \alpha^{3}}\right)-\operatorname{Er}\left(\frac{B_{64}}{8 \alpha^{2}}\right.\right. \\
& \left.\left.-\frac{\left(B_{72}+B_{74}\right)}{8 \alpha^{3}}+\frac{3 B_{77}}{16 \alpha^{4}}\right)\right] \\
& B_{31}=\operatorname{Pr}\left[\operatorname{Re} \frac{R_{84}}{4 \alpha^{2}}-\operatorname{Er}\left(\frac{B_{76}}{8 \alpha^{2}}\right.\right. \\
& \left.\left.-\frac{\left(B_{73}+B_{75}\right)}{4 \alpha^{3}}\right)\right] \\
& B_{32}=-\operatorname{Pr} \operatorname{Er} \frac{B_{77}}{8 \alpha^{2}}, \\
& B_{33}=\operatorname{Pr}\left[\operatorname{Re}\left(\frac{B_{81}}{4 \alpha^{2}}-\frac{R_{34}}{4 \alpha^{3}}\right)-\operatorname{Er}\left(\frac{R_{13}+R_{15}}{8 \alpha^{2}}\right.\right. \\
& \left.\left.-\frac{R_{26}}{8 \alpha^{3}}+\frac{3 R_{25}+2 R_{23}}{16 \alpha^{4}}\right)\right] \\
& B_{34}=\operatorname{Pr}\left[\operatorname{Re} \frac{B_{82}}{4 \alpha^{2}}-\operatorname{Er}\left(\frac{B_{72}+B_{74}}{8 \alpha^{2}}-\frac{B_{77}}{4 \alpha^{3}}\right)\right], \\
& B_{21}=\operatorname{Pr}\left[\frac{\operatorname{Re} B_{78}}{2}-\frac{\operatorname{Er}\left(B_{63}-B_{65}\right)}{4}\right] \text {, } \\
& B_{35}=-\operatorname{Pr} \operatorname{Er} \frac{\left(B_{73}+B_{75}\right)}{8 \alpha^{2}},
\end{aligned}
$$




$$
\begin{aligned}
& B_{36}=\operatorname{Sc}\left[\operatorname{Re} \frac{A_{57}}{4 \alpha^{2}}-\operatorname{Sr} B_{31}\right] \\
& B_{53}=-\mathrm{SrSc} B_{35}, \\
& B_{37}=\operatorname{Pr}\left[\frac{2 \operatorname{Re}\left(B_{93}+B_{94}\right)-\operatorname{Er}\left(B_{68}+B_{71}\right)}{36 \alpha^{2}}\right] \text {, } \\
& B_{54}=\operatorname{Sc}\left[\operatorname{Re} \frac{A_{55}}{4 \alpha^{2}}-\operatorname{Sr} B_{34}\right], \\
& B_{38}=B_{23} h_{1}^{4}+\sinh \alpha h_{1}\left(B_{24}+B_{25} h_{1}\right. \\
& \left.+B_{26} h_{1}^{2}\right)+\cosh \alpha h_{1}\left(B_{27}+B_{28} h_{1}\right. \\
& \left.+B_{29} h_{1}^{2}\right)+\sinh 2 \alpha h_{1}\left(B_{36}+B_{37} h_{1}\right. \\
& \left.+B_{38} h_{1}^{2}\right)+\cosh 2 \alpha h_{1}\left(B_{39}+B_{40} h_{1}\right. \\
& \left.+B_{41} h_{1}^{2}\right)+B_{21} h_{1}^{2}+B_{22} h_{1}^{3} \\
& +B_{36} \sinh 3 \alpha h_{1}+B_{37} \cosh 3 \alpha h_{1}, \\
& B_{39}=\frac{h_{1} B_{92}-h_{2} B_{91}}{h_{2}-h_{1}}, \quad B_{40}=\frac{B_{56}-B_{55}}{h_{2}-h_{1}}, \\
& B_{41}=-\operatorname{SrSc} B_{21}+\operatorname{ReSc} \frac{A_{51}}{2}, \\
& B_{42}=-\operatorname{SrSc} B_{22}+\operatorname{ReSc} \frac{B_{53}}{6}, \\
& B_{43}=-\operatorname{SrSc} B_{23}+\operatorname{ReSc} \frac{A_{54}}{12}, \\
& B_{44}=\operatorname{Sc}\left[\operatorname { R e } \left(\frac{6 A_{60}}{\alpha^{4}}-\frac{2 A_{62}}{\alpha^{3}}+\frac{A_{58}}{\alpha^{2}}\right.\right. \\
& \left.\left.+\frac{\left(A_{65}-A_{64}\right)}{2 \alpha^{2}}\right)-\operatorname{Sr} A_{67}\right], \\
& B_{45}=\operatorname{Sc}\left[\operatorname{Re}\left(\frac{B_{34}}{\alpha^{2}}-\frac{B_{37}}{\alpha^{3}}\right)-\operatorname{Sr} B_{25}\right] \text {, } \\
& B_{46}=\operatorname{Sc}\left[\operatorname{Re} \frac{A_{60}}{\alpha^{2}}-\operatorname{Sr} B_{26}\right] \text {, } \\
& B_{47}=\operatorname{Sc}\left[\operatorname { R e } \left(\frac{6 A_{63}}{\alpha^{4}}-\frac{2 A_{59}}{\alpha^{3}}+\frac{A_{61}}{\alpha^{2}}\right.\right. \\
& \left.\left.+\frac{\left(A_{65}-A_{64}\right)}{2 \alpha^{2}}-\frac{A_{57}}{4 \alpha^{3}}\right)-\operatorname{Sr} B_{27}\right], \\
& B_{48}=\operatorname{Sc}\left[\operatorname{Re}\left(-\frac{4 A_{60}}{\alpha^{3}}+\frac{A_{62}}{\alpha^{2}}\right)-\operatorname{Sr} B_{8}\right] \text {, } \\
& B_{49}=\operatorname{Sc}\left[\operatorname{Re} \frac{A_{63}}{\alpha^{2}}-\operatorname{Sr} B_{27}\right] \text {, } \\
& B_{50}=\mathrm{Sc}\left[\operatorname{Re}\left(\frac{A_{56}}{4 \alpha^{2}}-\frac{A_{55}}{4 \alpha^{3}}\right)-\operatorname{Sr} B_{30}\right] \text {, } \\
& B_{51}=-\operatorname{SrSc} B_{32}, \quad B_{52}=\operatorname{Sc}\left[\operatorname{Re} \frac{A_{52}}{4 \alpha^{2}}-\operatorname{Sr} B_{33}\right], \\
& B_{55}=\operatorname{Sc}\left[\operatorname{Re} \frac{\left(A_{63}+A_{67}\right)}{18 \alpha^{2}}-\operatorname{Sr} B_{36}\right] \text {, } \\
& B_{56}=\operatorname{Sc}\left[\operatorname{Re} \frac{\left(A_{64}+A_{65}\right)}{18 \alpha^{2}}-\operatorname{Sr} B_{37}\right] \text {, } \\
& B_{57}=-\frac{3 A_{19}}{2 \alpha^{3}}+\frac{A_{17}}{2 \alpha^{2}}+A_{23} \alpha^{2}, \\
& B_{58}=-\frac{A_{20}}{8 \alpha^{2}}+\frac{A_{18}}{4 \alpha}, \quad B_{59}=\frac{A_{19}}{8 \alpha}, \\
& B_{60}=-\frac{3 A_{20}}{2 \alpha^{3}}+\frac{A_{18}}{2 \alpha^{2}}+A_{24} \alpha^{2}, \\
& B_{61}=-\frac{A_{19}}{8 \alpha^{2}}+\frac{A_{17}}{4 \alpha}, \quad B_{62}=\frac{A_{20}}{8 \alpha}, \quad B_{63}=-\frac{A_{16}}{2 \alpha^{2}}, \\
& B_{64}=4 A_{3} \alpha^{2} A_{15}+\kappa\left(-2 A_{1} A_{3} A_{3}^{\prime} \alpha^{4}\right. \\
& \left.+2 A_{2}^{\prime} A_{3} A_{4} \alpha^{5}+6 A_{1}^{\prime} A_{3}^{2} \alpha^{4}-6 A_{3} A_{4}^{\prime} \alpha^{3}\right), \\
& B_{65}=4 A_{3} \alpha^{2} B_{59}+4 A_{4} \alpha B_{56}+\kappa\left(-2 A_{1} A_{3} A_{4}^{\prime} \alpha^{4}\right. \\
& \left.-2 A_{3}^{\prime} A_{1} A_{4} \alpha^{4}+2 A_{2}^{\prime} A_{3}^{\prime} \alpha^{5}+2 A_{4}^{2} A_{2}^{\prime} \alpha^{5}\right), \\
& B_{66}=4 A_{4} \alpha^{2} B_{59}+\kappa\left(-2 A_{1} A_{4} A_{4}^{\prime} \alpha^{4}\right. \\
& \left.+2 A_{2}^{\prime} A_{3} A_{4} \alpha^{5}+6 A_{1}^{\prime} A_{4}^{2} \alpha^{4}-6 A_{4} A_{3}^{\prime} \alpha^{3}\right), \\
& B_{67}=4 A_{4} \alpha^{2} B_{62}-6 \kappa A_{1}^{\prime} A_{4} \alpha^{2}, \\
& B_{68}=4 A_{4} \alpha^{2} B_{62}-6 \kappa A_{1}^{\prime} A_{3} \alpha^{2}, \\
& B_{69}=6 \kappa A_{3}^{\prime} A_{4}^{2} \alpha^{5}, \quad B_{70}=6 \kappa A_{4}^{\prime} A_{3}^{2} \alpha^{5} \text {, } \\
& B_{71}=12 \kappa A_{3} A_{3}^{\prime} A_{4} \alpha^{5}+6 \kappa A_{4}^{\prime} A_{4}^{2} \alpha^{5}, \\
& B_{72}=12 \kappa A_{3} A_{4}^{\prime} A_{4} \alpha^{5}+6 \kappa A_{3}^{\prime} A_{3}^{2} \alpha^{5}, \\
& B_{73}=4 A_{3} \alpha^{2} R_{7}+2 \kappa A_{1}^{\prime} A_{3} A_{4} \alpha^{5}, \\
& B_{74}=4 A_{3} \alpha^{2} B_{58}, \quad B_{75}=4 A_{4} \alpha^{2} B_{60}+2 \kappa A_{1}^{\prime} A_{3} A_{4} \alpha^{5}, \\
& B_{76}=4 A_{4} \alpha^{2} B_{61}, \\
& B_{77}=4 A_{3} \alpha^{2} B_{60}+4 A_{4} \alpha^{5} B_{57} \\
& +2 \kappa A_{1}^{\prime} A_{3}^{2} \alpha^{5}+2 \kappa A_{1}^{\prime} A_{4}^{2} \alpha^{5}, \\
& B_{78}=4 A_{3} \alpha^{2} R_{11}+4 A_{4} \alpha^{5} B_{58} \text {, } \\
& B_{79}=A_{1} A_{5}^{\prime}-A_{2}^{\prime} A_{6},
\end{aligned}
$$




$$
\begin{aligned}
& B_{80}=A_{1} A_{6}^{\prime}-A_{1}^{\prime} A_{6}-2 A_{2}^{\prime} A_{7}, \\
& B_{81}=-2 A_{1}^{\prime} A_{7}+A_{1} A_{7}^{\prime}, \\
& B_{82}=A_{1} A_{8}^{\prime}-2 A_{2}^{\prime} A_{9} \alpha, \\
& B_{83}=-2 A_{1}^{\prime} A_{9} \alpha, \quad B_{84}=A_{1} A_{8}^{\prime}-2 A_{2}^{\prime} A_{9} \alpha, \\
& B_{85}=-2 A_{1}^{\prime} A_{8} \alpha, \quad B_{86}=A_{3} A_{5}^{\prime} \alpha-A_{4}^{\prime} A_{6}, \\
& B_{87}=A_{3} A_{6}^{\prime} \alpha-2 A_{4}^{\prime} A_{7}, \quad B_{88}=A_{3} A_{7}^{\prime} \alpha, \\
& B_{89}=A_{4} A_{1}^{\prime} \alpha-A_{3}^{\prime} A_{6}, \quad B_{90}=A_{4} A_{6}^{\prime} \alpha-2 A_{3}^{\prime} A_{7}, \\
& B_{91}=A_{4} A_{8}^{\prime} \alpha, \quad B_{92}=A_{3} A_{9}^{\prime} \alpha-2 A_{4}^{\prime} A_{8} \alpha, \\
& B_{93}=A_{4} A_{8}^{\prime} \alpha-2 A_{3}^{\prime} A_{9} \alpha, \\
& B_{94}=A_{3} A_{8}^{\prime} \alpha-2 A_{4}^{\prime} A_{9} \alpha, \\
& B_{95}=A_{4} A_{9}^{\prime} \alpha-2 A_{3}^{\prime} A_{8} \alpha,
\end{aligned}
$$

where prime denotes the derivative with respect to $x$ :

$$
\begin{aligned}
\alpha= & \frac{M \cos \gamma}{\sqrt{2}}, \\
C_{1}= & \cosh \alpha h_{1}-\cosh \alpha h_{2}, \\
C_{2}= & \sinh \alpha h_{1}-\sinh \alpha h_{2}, \\
C_{3}= & \cosh \alpha h_{1}+\cosh \alpha h_{2}, \\
C_{4}= & \sinh \alpha h_{1}+\sinh \alpha h_{2}, \\
C_{5}= & A_{2}+2 \alpha \beta A_{3}, \quad C_{6}=A_{1}+2 \alpha \beta A_{4}, \\
C_{7}= & \frac{C_{6} C_{1}-C_{2} C_{5}}{\left(h_{2}-h_{1}\right) C_{5}} \\
& -\frac{C_{6}\left(\alpha \sinh \alpha h_{1}+2 \alpha^{2} \beta \cosh \alpha h_{1}\right)}{C_{5}} \\
C_{9}= & \frac{C_{6} \cosh \alpha h_{2}}{C_{5}}-\sinh \alpha h_{2}-\frac{h_{2}\left(C_{6} C_{1}-C_{2} C_{5}\right)}{\left(h_{2}-h_{1}\right) C_{5}} \\
& +\alpha \cosh \alpha h_{1}+2 \alpha^{2} \beta \sinh \alpha h_{1}, \\
C_{8}= & \frac{C_{6} \cosh \alpha h_{1}}{C_{5}}-\sinh \alpha h_{1}-\frac{h_{1}\left(C_{6} C_{1}-C_{2} C_{5}\right)}{\left(h_{2}-h_{1}\right) C_{5}} \\
& \\
&
\end{aligned}
$$

\section{Biographies}

Ambreen Afsar Khan received her $\mathrm{PhD}$ degree from the Department of Mathematics, Quaid-i-Azam University, Islamabad, Pakistan. She is an Assistant Professor at International Islamic University, Islamabad, Pakistan. Her several research articles in the field of fluid mechanics have been published in the journals of international repute.

Hafsa Usman is working as a Research Scholar under the supervision of Dr. Ambreen Afsar Khan in the Department of Mathematics and Statistics, International Islamic University, Islamabad, Pakistan.

Kambiz Vafai is Professor of Mechanical Engineering at University of California, Riverside (UCR), where he started as the Presidential Chair in the Department of Mechanical Engineering. He joined UCR from The Ohio State University, where he received outstanding research awards as Assistant, Associate, and Full Professor. He is a fellow of American Association for Advancement of Science, American Society of Mechanical Engineers, and World Innovation Foundation and an Associate Fellow of the American Institute of Aeronautics and Astronautics. He is in the ISI highly cited category. He has carried out various sponsored research projects through companies, governmental funding agencies, and national labs. He has also consulted in various companies and national labs and has been granted seven US patents. He was the recipient of the ASME Classic Paper Award in 1999 and received the 2006 ASME Heat Transfer Memorial Award. He is also the recipient of the highest award of the International Society of Porous Media. Dr. Vafai received his BS degree from the University of Minnesota Minneapolis, and the MS and PhD degrees from the University of California, Berkeley.

Rahmat Ellahi is Professor of Mathematics in the Faculty of Science at Taibah University, Madinah Munawwarah, Kingdom of Saudi Arabia. He is Fulbright Fellow at the University of California, Riverside, USA. He received outstanding awards such as Fulbright Award of USA, Best University Teacher Award from Higher Education Commission of Pakistan, Valued Reviewer from Elsevier, Best Book Award, etc. He is editor of 11 international journals and is on review panels of more than 175 international journals. He has published 135 research articles in journals of international repute. He has supervised $5 \mathrm{PhD}$ students and several research students at MS level. He has carried out 2 sponsored research projects through HEC under NRPU and USEFP funding. He is the author of 6 books. He has been contentiously honored with annual Research Productivity Award based on his excellent 
scientific achievements by Ministry of Science and Technology since 2009. Recently, PCST honored him with the 7th topmost Productive Scientist of Pakistan Award "Category A" in the year 2015. He has established international collaboration with 30 prominent scientists of his field. He has the honor of being the Founder Chairman of the Department of Mathematics at International Islamic University Islamabad (IIUI).
The Department of Statistics and Physics at IIUI has been successfully established due to his humble contribution. In addition, he is founder of Quality Enhancement Cell at IIUI. He is actively involved in different academic bodies at national and International levels. His field of research consisting of nanofluid, heat transfer, porous media, peristaltic motion, and blood flow has many industrial applications. 